\title{
Desenvolvimento do Aplicativo ML-SAI para Android com Uso do App Inventor
}

\author{
Ernane Rosa Martins , Luís Manuel Borges Gouveia² \\ ${ }^{1}$ Instituto Federal de Goiás (IFG) \\ Caixa Postal 72.811-580 - Luziânia - GO - Brasil \\ ${ }^{2}$ Universidade Fernando Pessoa (UFP) \\ Caixa Postal 4249-004 - Porto - Portugal \\ ernane.martins@ifg.edu.br, lmbgeufp.edu.pt
}

\begin{abstract}
The automation of a pedagogical model is currently indispensable, as it is freely available. This paper aims to report the development of the $M L$ SAI application through the App Inventor tool, based on the pedagogical model for e-learning m-learning activities, which was based on the Inverted Classroom (IC) theory. Contribute to guide the planning and implementation of teaching activities. The research is characterized as exploratory, as it addresses new problems still little explored. The app can help guide the planning and implementation of teaching and learning activities using mobile devices.
\end{abstract}

Resumo. A automatização de um modelo pedagógico atualmente é indispensável, por a disponibilização deste gratuitamente. Este trabalho tem como objetivo relatar o desenvolvimento do aplicativo ML-SAI por meio da ferramenta App Inventor, com base no modelo pedagógico para atividades de m-learning de mesmo nome, que foi fundamentado na teoria da Sala de Aula Invertida (SAI), visando contribuir para orientar o planejamento e a realização de atividades de ensino. A pesquisa realizada caracteriza-se como exploratória, por abordar novos problemas ainda pouco explorados. $O$ aplicativo poderá contribuir para orientar o planejamento e a realização de atividades de ensino e aprendizado com o uso de dispositivos móveis.

\section{Introdução}

Para Freeman et al. (2014) a aprendizagem ativa pode aumentar o desempenho dos alunos. Porem muitos professores tentam encontrar formas de incorpora-las na sala de aula. Uma estratégia que se tornou popular atualmente é o modelo de Sala de Aula Invertida (SAI), em que os alunos aprendem os conteúdos antes da aula por meio das tecnologias digitais e realizam atividades práticas tais como: projetos, experimentos, debates, atividades em grupo e pesquisas, durante a aula tirando suas dúvidas e compartilhando seus aprendizados, com o auxílio do professor (Mattar, 2017).

Behar, Passerino \& Bernardi (2007, p.3) descrevem os modelos como sendo "um sistema figurativo que reproduz a realidade de forma mais abstrata, quase esquemática, e que serve de referência”. Os modelos pedagógicos são erroneamente definidos na literatura como sinônimos de estratégias de ensino, metodologias de ensino, currículo ou 
como teoria de aprendizagem. A definição adotada neste estudo é de que o modelo pedagógico é "um sistema de premissas teóricas que representa, explica e orienta a forma como se aborda o currículo e que se concretiza nas práticas pedagógicas e nas interações professor-aluno-objeto de conhecimento" (Behar; Passerino \& Bernardi, 2007, p. 4).

O modelo pedagógico segundo Behar (2009), é constituído basicamente por dois elementos: a Arquitetura Pedagógica (AP), que é a estrutura principal; e as estratégias para a aplicação da mesma, que são as dinâmicas do modelo pedagógico, que dependem do contexto e variáveis que envolvem o processo educativo. O ML-SAI é um modelo pedagógico construído a partir de estudos exploratórios, utilizando os conceitos de sala de aula invertida combinada com alguma tecnologia móvel, como Kahoot, WhatsApp e Facebook. Estas investigações permitiram encontrar novas possibilidades e potencialidades da utilização da Teoria da Sala de Aula Invertida com o auxílio de Tecnologia Móvel (Martins \& Gouveia, 2019).

A popularidade dos aplicativos móveis cresceu significativamente nos últimos anos, simplesmente pelo fato destes aplicativos serem executados em dispositivos móveis, principalmente nos smartphones se tornaram praticamente onipresentes. Para sua utilização basta simplesmente baixa-los on-line, no App Store da Apple ou Google Play for Android Apps (Jabangwe et al., 2018). Os aplicativos para dispositivos móveis são softwares com objetivos específicos, destinados a facilitar o desempenho de atividades de diversas finalidades, como por exemplo o modelo pedagógico ML-SAI. Estes aplicativos apresentam como vantagem: a facilidade de uso, melhorando a experiência com o uso de recursos e interface dos dispositivos, otimizando a navegação e a agilizando as ações; menor custo de acesso, a interface é adaptada para o dispositivo, o tráfego de dados necessários para navegação é muito menor se comparado ao uso de navegadores convencionais; melhor uso dos recursos que o aparelho possui, tais como: GPS, câmera fotográfica, bluetooth, entre outros; e acesso off-line, permitindo armazenar informações no próprio aparelho (Porto, 2012).

A ferramenta App Inventor utilizada neste presente trabalho foi criada pela Google e melhorada pelo Instituto de Tecnologia de Massachusetts, atualmente está vem ganhando destaque por oferece uma nova forma de programar para o Sistema Operacional Android, utilizando para isto diversos blocos de códigos prontos, sendo uma forma muito mais intuitiva e ágil de desenvolvimento de software, como se fosse um quebra-cabeça que vai sendo montado através de arrastar e soltar (Hardesty, 2010).

Neste contexto, o objetivo deste presente trabalho é relatar o desenvolvimento do aplicativo ML-SAI por meio da ferramenta App Inventor, com base no modelo pedagógico para atividades de m-learning de mesmo nome, que foi fundamentado na teoria da Sala de Aula Invertida (SAI), visando contribuir para orientar o planejamento e a realização de atividades de ensino.

\section{Apresentação do ML-SAI}

O modelo pedagógico ML-SAI fornece algumas sugestões de estratégias a professores e pesquisadores interessados em utiliza-lo, orientando estes no desenvolvimento de atividades de m-learning. O ML-SAI apresenta uma Arquitetura Pedagógica (AP) reestruturada, levando em consideração os conceitos da Sala de Aula Invertida, os 
aspectos relacionados a utilização dos dispositivos móveis e os estudos exploratórios realizados durante as pesquisas realizadas para sua construção.

A AP foi definida em seis aspectos: contexto, normatização, papeis, tecnologias, ações e limitações. A AP e as estratégias para a Aplicação da AP estão apresentadas na Tabela 1.

Tabela 1. Modelo pedagógico proposto: ML-SAl (Martins \& Gouveia, 2019)

\begin{tabular}{|c|c|}
\hline $\begin{array}{l}\text { Arquitetura } \\
\text { Pedagógica } \\
\text { (AP) }\end{array}$ & Estratégias para a Aplicação da AP \\
\hline Contexto & $\begin{array}{l}\text { Definir os objetivos e motivos das atividades e ações proposta, deixando-os claros para } \\
\text { todos os envolvidos; } \\
\text { Identificar os instrumentos, recursos, características das atividades e ações, dos alunos } \\
\text { e do curso; }\end{array}$ \\
\hline Normatização & $\begin{array}{l}\text { Organizar regras e procedimentos para orientar as ações e interações; } \\
\text { Estabelecer normas para utilização dos dispositivos móveis (quando utilizar, qual a } \\
\text { finalidade, etc.); }\end{array}$ \\
\hline Papeis & $\begin{array}{l}\text { Compreender o papel do aluno no processo de aprendizagem, suas motivações, } \\
\text { interesses e habilidades; } \\
\text { Entender o papel do professor como condutor e facilitador da aprendizagem; }\end{array}$ \\
\hline Tecnologias & $\begin{array}{l}\text { Definir os dispositivos móveis, aplicativos e recursos tecnológicos que serão } \\
\text { utilizados, considerando as características físicas, técnicas e funcionais dos mesmos, } \\
\text { tais como: ambiente virtual, Sílabe, Moodle, Facebook, Khan Academy, YouTube, } \\
\text { vídeo-aula, músicas, slides, fotografias, áudios, textos, entre outros, estabelecendo } \\
\text { prioridade para aplicativos livres e gratuitos; } \\
\text { Verificar a necessidade e disponibilidade de conexão com a Internet; }\end{array}$ \\
\hline Ações & $\begin{array}{l}\text { Especificar se as ações serão individuais, em grupo ou ambas, se estas serão comuns a } \\
\text { todos os alunos ou diferenciadas por aluno ou grupo de alunos; } \\
\text { Definir ferramentas de comunicação e sistemas de apoio para dar suporte aos alunos } \\
\text { em caso de dificuldades; } \\
\text { Definir se as ações serão realizadas em um mesmo local, ao mesmo tempo ou em } \\
\text { locais e momentos distintos; } \\
\text { Estabelecer práticas educacionais favoráveis ao aprendizado (situações problemas, } \\
\text { aplicações práticas, colaborativas, autônomas, críticas, em contextos reais, pesquisas), } \\
\text { levando em consideração os ambientes de aprendizagem (on-line, salas de aula, } \\
\text { laboratórios) de preferência com os dispositivos móveis dos próprios alunos; } \\
\text { Incentivar a interação entre os alunos e com o professor, por meio do uso de } \\
\text { dispositivos móveis, com foco no desenvolvimento da atividade proposta; } \\
\text { Determinar os mecanismos de avaliação de desempenho e certificação da } \\
\text { aprendizagem, se individuais ou em equipes, de preferência continua, e disponibiliza- } \\
\text { los para os alunos; } \\
\text { Estabelecer momentos de reflexão e análise das atividades realizadas, buscando } \\
\text { colaborar na melhoria continua de novas atividades; } \\
\text { Estruturar os conteúdos que serão disponibilizados em ambiente virtual, para que os } \\
\text { alunos possam acessa-los por meio de um dispositivo móvel, quando e quantas vezes } \\
\text { quiserem, se possível com o acompanhamento das visualizações pelo professor; } \\
\text { Realizar uma curadoria dos conteúdos já existentes na Internet, por meio de } \\
\text { plataformas como Khan Academy e o YouTube em busca de bons vídeos educativos, ou } \\
\text { caso não sejam encontrados, gravar vídeos ou áudios utilizando as ferramentas que } \\
\text { existem no próprio dispositivo móvel; } \\
\text { Estimular diferentes formas de aprendizado por meio de diferentes fontes de conteúdo, } \\
\text { tais como: vídeos, áudios, imagens, textos, slides, questões, entre outras; } \\
\text { Elaborar um roteiro de atividades do que será feito dentro da sala de aula, de modo a } \\
\text { otimizar o tempo em sala de aula, utilizando projetos, trabalhos ou solução de }\end{array}$ \\
\hline
\end{tabular}




\begin{tabular}{|l|l|}
\hline \multirow{3}{*}{ Limitações } & problemas, que se conectem com o que foi visto previamente na plataforma; \\
\hline & $\begin{array}{l}\text { Levantar os principais pré-requisitos das atividades e possíveis distratores do } \\
\text { aprendizado; } \\
\text { Identificar quais conteúdos podem ser melhor trabalhados com tecnologias móveis; } \\
\text { Verificar se os materiais pedagógicos podem ser utilizados em dispositivos móveis, } \\
\text { considerando tamanho da tela, usabilidade, capacidade de armazenamento e modelos } \\
\text { de dispositivos diferentes; } \\
\text { Verificar a disponibilidade de dispositivos móveis, tomadas para recarregar as baterias } \\
\text { dos celulares, conexão com a Internet, quando necessário, e se os aplicativos } \\
\text { apresentam interface adequada a aprendizagem do conteúdo. }\end{array}$ \\
\hline
\end{tabular}

A estrutura do modelo pedagógico ML-SAI apresentado na Tabela 1, foi formatada para que se possa adaptar e colaborar com o desenvolvimento de diversas atividades de m-learning envolvendo diferentes conteúdos e dispositivos móveis, levando em consideração principalmente as características especificas dos alunos.

\section{Métodos e procedimentos}

O aplicativo foi desenvolvido com o emprego do software livre App Inventor. Escolhido por ser um ambiente de programação visual de código aberto, que possibilita a criação de aplicativos para Android com conhecimentos básicos de programação, funcionando online possibilitando a codificação por meio de blocos de códigos prontos e montáveis

Os procedimentos utilizados para a pesquisa e desenvolvimento deste trabalho é de caráter exploratório, com o desenvolvimento de pesquisa bibliográfica, buscando alinhar os fatores teóricos ao projeto prático, com o desenvolvimento de atividade prática de elaboração do aplicativo ML-SAI. A presente pesquisa é classificada como exploratória pois segundo Brown (2006) este tipo de estudo busca abordar novos problemas sobre os quais pouca ou nenhuma pesquisa anterior foi feita. Para tal, foram definidas as etapas de criação do aplicativo conforme Beltrame et al. (2017), com a concepção das ideias, modelagem da solução, desenho das telas, programação, revisão, testes e publicação do aplicativo na plataforma de distribuição.

\section{Implementação do ML-SAI}

Após a concepção do modelo pedagógico, este foi implementado em versão digital, como forma de facilitar o acesso por parte de professores e pesquisadores. A Figura 1 apresenta a tela principal desta versão para ilustrar e auxiliar a explicação do mesmo. 


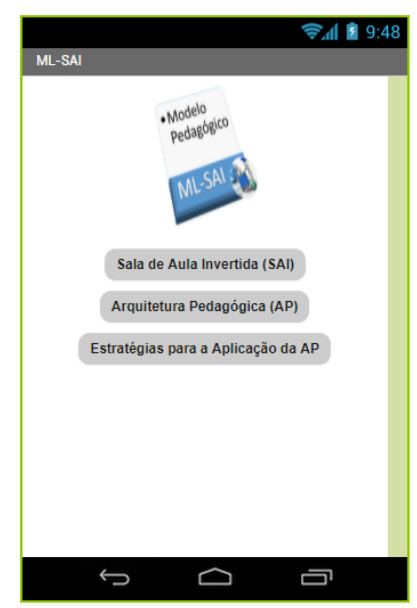

Figura 1. Tela inicial da versão digital do ML-SAl.

$\mathrm{Na}$ Figura temos os botões que direcionam para novas as telas que mostram os conceitos de Sala de Aula Invertida, Arquitetura Pedagógica (AP) e as Estratégias para a Aplicação da AP, sendo estes conceitos definidos anteriormente neste trabalho. A tela sobre as Estratégias para a Aplicação da AP é apresentada na Figura 2, e direciona para o detalhamento da AP estabelecida em seus seis aspectos: contexto, normatização, papeis, tecnologias, ações e limitações.

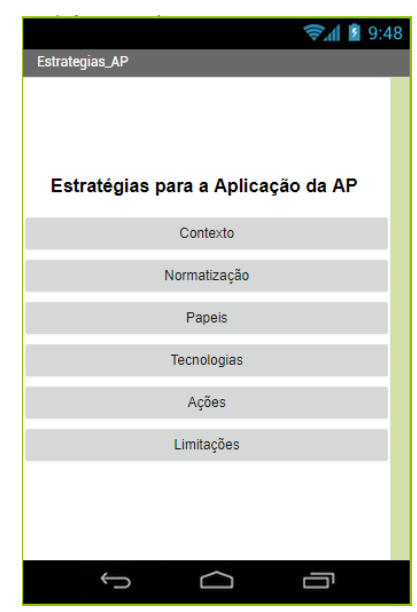

Figura 2. Tela sobre as Estratégias para a Aplicação da AP

Assim, em cada componente há um link que remete à explicação sobre o mesmo, sendo estas apresentadas anteriormente neste trabalho na Tabela 1. O desenvolvimento desta versão digital do modelo pedagógico ML-SAI, poderá colaborar com o seu amplo conhecimento, de modo a possibilitar a orientação de uma quantidade maior de profissionais no desenvolvimento de práticas educacionais diversas que envolvam $m$ learning, tais como: disciplinas, minicursos e atividades especificas.

\section{Conclusões}

O objetivo deste trabalho é relatar o desenvolvimento do aplicativo ML-SAI por meio da ferramenta App Inventor, com base no modelo pedagógico para atividades de $m$ - 
learning de mesmo nome, que foi fundamentado na teoria da Sala de Aula Invertida (SAI). Este será disponibilizado gratuitamente, e poderá contribuir para orientar o planejamento e a realização de atividades de ensino e aprendizado por meio de dispositivos móveis. A ferramenta App Inventor foi de fácil utilização, mostrando-se bastante versátil e flexível durante o processo de desenvolvimento da aplicação, por ser em português, com diversas funções simples e complexas de desenvolvimento disponíveis, não apresentou nenhum tipo de problema de travamento ou de execução, e por possibilitar uma codificação ágil e eficaz.

Com o desenvolvimento do aplicativo ML-SAI, espera-se contribuir significativamente para a melhoria do processo de ensino-aprendizagem, por meio de uma nova abordagem que estimule a incorporação de recursos computacionais móveis nas atividades de sala de aula e na promoção da aprendizagem de forma geral. A próxima etapa a ser realizada nos próximos meses, será a disponibilização do aplicativo ML-SAI para a comunidade acadêmica, e realizar novos estudos verificando a necessidade de possíveis adaptações do mesmo.

\section{Referências}

Behar, P. A. (2009). Modelos pedagógicos em educação a distância. 311 p. Porto Alegre: Artmed.

Behar, P. A.; Passerino, L. and Bernardi, M. (2007). Modelos Pedagógicos par a Educação a Distância: pressupostos teóricos para a construção de objetos de aprendizagem. Revista Novas Tecnologias na Educação, Porto Alegre, v.5, n. 2, p. 1 - 12.

Beltrame, W. A. R.; Baeta, K. D. S.; Marcos, R. de S.; Piassi, D. F. S. D.; Reboli, G. da S.; Sezini, S. L. M. and Favatto, T. S. (2017). Aventura Matemática: Demonstração e Avaliação de um Aplicativo para Aprendizagem de Operações Básicas. In: VI Congresso Brasileiro de Informática na Educação. p. 397-400.

Brown, R. B. (2006). Doing your Dissertation in Business and Management: The Reality of Researching and Writing. SAGE, London.

Freeman, S., Eddy, S. L., McDonough, M., Smith, M. K., Okoroafor, N., Jordt, H., and Wenderoth, M. P. (2014). Active learning increases student performance in science, engineering, and mathematics. PNAS, v. 111, n. 23, p. 8410-8415.

Hardesty, L. (2010). The MIT roots of Google's new software. 19/08/2010. Disponível em: http://news.mit.edu/2010/android-abelson-0819. Acessado em: 14/08/2019.

Jabangwe, R.; Edisonb, H. and Ducc, A. N. (2018). Software engineering process models for mobile app development: A systematic literature review. The Journal of Systems \& Software, v. 145, p. 98-111.

Martins, E. R. and Gouveia, L. M. B. (2019). ML-SAI: Um Modelo Pedagógico para Atividades de M-Learning que Integra a Abordagem da Sala de Aula Invertida. In: XXVII Workshop Sobre Educação em Computação (WEI-SBC), v. 27, Belém. p. 121-130.

Mattar, J. (2017). Metodologias Ativas: para a educação presencial, blended e a distância. 1 ed. São Paulo: Artesanato Educacional, 118p.

Porto, F. (2012). Aplicativos Mobile: Definições, História E Previsões. Disponível em: http://tectriadebrasil.com.br/blog/mercado-de-midias-sociais-blog/aplicativosmobiledefinicoes-historia-e-previsoes/. Acessado em: 14/08/2019. 